\title{
JACOB STURM.
}

\section{REDE}

GEHALTEN BEI ÜBERNAHME DES RECTORATS DER UNIVERSITÄT STRASSBURG AM 1. MAI $18 \overline{6}$.

vox

\section{HERMANN BAUMGARTEN.}

STRA SSBURG.

K A RL J. TRÜB N ER

LONDON.

TRÜBNER \& COMP 1876. 
Buchdruckerei von $\mathrm{G} O \mathrm{t}^{\mathrm{to}}$ in Darmstadt. 